\title{
ANALYSIS OF HUMAN BREATH SAMPLES USING A MODIFIED THERMAL DESORPTION - GAS CHROMATOGRAPHY ELECTROSPRAY IONIZATION INTERFACE.
}

\author{
1James C. Reynolds, ${ }^{1}$ Modupe A. Jimoh, ${ }^{1}$ Cristina Guallar-Hoyas, ${ }^{1}$ Colin S. Creaser, \\ 2Salman Siddiqui, ${ }^{1}$ C.L. Paul Thomas \\ ${ }^{1}$ Centre for Analytical Science, Department of Chemistry, \\ Loughborough University, Ashby Road, Loughborough, LE11 3TU UK \\ 2NIHR Leicester Respiratory Biomedical Research Unit \\ Glenfield Hospital, Groby Road, Leicester, LE3 9QP
}




\section{Abstract}

A two-stage thermal desorption/secondary electrospray ionisation/time-of-flight mass spectrometry for faster targeted breath profiling has been studied. A new secondary electrospray ionisation (SESI) source was devised to constrain the thermal desorption plume and promote efficient mixing in the ionization region. Further a chromatographic pre-separation stage was introduced to suppress interferences from siloxanes associated with thermal desorption profiles of exhaled breath samples.

In-vitro tests with 5-nonanone indicated an increased sensitivity and a lowered limit-ofdetection, both by a factor of $\sim 4$, the latter to an on-trap mass of $14.3 \mathrm{ng}$, equivalent to a sampled breath concentration of $967 \mathrm{ppt}_{\mathrm{v}}$, Analysis of the mass spectrometric responses from 20 breath samples acquired sequentially from a single participant indicated enhanced reproducibility (reduced relative standard deviations (RSD) for 5-nonanone, benzaldehyde and 2-butanone were $28 \%, 16 \%$ and 14\% respectively. The corresponding values for an open SESI source were that 5-nonanone was not detected, with \%RSD of $39 \%$ for benzaldehyde and $31 \%$ for 2-butanone.)

The constrained source with chromatographic pre-separation resulted in an increase in the number of detectable VOC from 260 mass spectral peaks with an open SESI source to 541 peaks with the constrained source with pre-separation. Most of the observed VOC were present at trace levels, at less than $2.5 \%$ of the intensity of the base peak.

Seventeen $2.5 \mathrm{dm}^{3}$ distal breath samples were collected from asthma patients and healthy controls respectively, and subjected to comparative high-throughput screening using thermal desorption/SESI/time-of-flight mass spectrometry (TD-SESI-ToFMS). Breath metabolites were detected by using a background siloxane ion (Hexamethylcyclotrisiloxane $m / z$ 223.0642) as an internal lockmass. 11 breath metabolites were selected from the breath research literature and successfully targeted. These data reinforce the proposition that TD-SESI-MS has potential for development as a rapid screening method for disease stratification and targeted metabolism profiling. 


\section{Introduction}

In 1986 Fenn et al. noted the efficient electrospray ionisation (ESI) of volatile organic compounds (VOC) ${ }^{1,2}$ Hill et al. subsequently interfaced an ESI source to an ion mobility spectrometer, ${ }^{3}$ and went on to show how VOC product ions could be generated by ESI for ion mobility spectrometry (IMS) determination and used the term secondary electrospray ionisation (SESI) ${ }^{4}$ to describe this process. The potential sensitivity of SESI was evident in the early descriptions of SESI with Hill and co-workers reporting a well-defined response $(\mathrm{s} / \mathrm{n}>20)$ for cocaine from a mass flux of ca. $1 \mathrm{pg} \cdot \mathrm{s}^{-1}$.

In SESI-IMS the electrospray plume was directed into the reaction region of an ion mobility spectrometer that contained sampled narcotic vapours. The high-sensitivity of SESI was combined with a quadrupole-time-of-flight mass spectrometer (ToFMS), operating in the negative mode, to profile volatile fatty acids in exhaled breath samples. ${ }^{5}$ Here, saturated and unsaturated fatty acids ranging from C3 to C14 in length were isolated at estimated concentrations in the order of 100 ppt $_{\mathrm{v}}$; indicative of a highsensitivity assay. More recently, volatile fatty acid profiling has been used to identify individuals from a cohort of 65 participants with a global recognition score of $63 \%$ (43 out of 65 correct) which suggests that this approach has the potential to distinguish and identify specific metabolic phenotypes. ${ }^{6}$

Similarly, Cooks et al. described extractive electrospray ionisation (EESI) ${ }^{7}$ based on mixing aerosol from a nebulised sample spray with an electrospray plume. The resultant interactions between electrospray droplets, ions and the aerosolised solution of analytes efficiently generated analyte ions. The potential utility of EESI was first demonstrated in a study that screened urine, surface waters and milk for trace-levels of pesticides and explosives. This study was a significant development in that proof-of-concept data were presented that highlighted high-speed quantitative assays of complex mixtures with substantial reductions in the resources, time and skill required to achieve high-fidelity analytical outcomes. The sensitivity of the approach and its tolerance of complex matrices was illustrated through the detection of 1 pmol of 1,3,5-trinitroperhydro-1,3,5triazine (RDX) spiked into undiluted mouse urine and $1 \mathrm{pmol}$ of trinitrotoluene (TNT) spiked into polluted river water; based on EESI analysis without sample clean-up. The direct detection of volatile potentially genotoxic impurities by thermal desorption EESI combined with MS and IMS-MS has also been reporte. ${ }^{8,9}$ Coupling a gas chromatograph to an electrospray mass spectrometer has also been accomplished adding in a 
hyphenated separation to resolve complex mixtures. This was demonstrated using a multiple channel ( 7 emitter) ESI source in $1998,{ }^{10}$ here the GC outlet was directed into the central electrospray emitter enabling eluted analytes to interact with the solvent before being electrosprayed. This novel approach was able to detect acylated aliphatic acids and was used to monitor a dehydrogenation of dimethylhydrazine in real-time. The combination of EESI-MS and atmospheric pressure chemical ionization (APCI-MS) with gas chromatography was used to detect a range of volatile organic compounds including pyridine, aminopyridine, n-vinylpyridine and the controlled substances 3,4methylenedioxy-N-methylamphetamine (MDMA) and 3,4-methylenedioxy-Nethylamphetamine (MDEA) by Brenner et. al. in 2008. ${ }^{11}$ Limits of detection for the pyridines were determined to be in the low picomole range indicating the sensitivity of the technique.

Breath profiling is an increasingly important approach to aid clinicians in diagnosing disease. Due to the non-invasive manner in which samples are collected, taking a breath sample can be part of a routine medical examination, giving it the potential to be used as a screening method for large populations. The importance of breath sampling and its capability to identify biomarkers for different diseases has been the subject of a number of review articles which highlight developments in clinical breath profiling. ${ }^{12-15}$ Breath sampling can be accomplished either offline using established lab-based techniques such as GC and GCXGC-ToFMS16 which can be coupled to preconcentration techniques such as thermal desorption to enable high resolution measurements on low concentration breath metabolites. The development of techniques such as proton transfer reaction-mass spectrometry (PTR-MS) ${ }^{17}$ and selected ion flow tube - mass spectrometry (SIFT-MS) ${ }^{18}$ has enabled monitoring of highly volatile species in real-time, providing diagnostic data in a very short timescale, however these techniques are less useful for the analysis of larger, less volatile species.

The extension of EESI-MS to exhaled breath profiling ${ }^{19-24}$ was a logical development since it enables faster analysis times than GC-MS, while maintaining the ability to detect semivolatile species solvated in aerosol particles. In 2007 EESI was used for breath-by-breath monitoring of exhaled urea concentration. ${ }^{19}$ In the same year EESI was used to ionise non-volatile components such as carbohydrates in breath, thereby bridging the previously disparate approaches of exhaled breath condensate analysis and exhaled breath 
analysis. ${ }^{20}$ In a study of the breath of smokers, nicotine was detected at $50 \mathrm{pg} \mathrm{m}^{-3}$ using EESI. ${ }^{21}$ Translational research has used SESI-MS to monitor the pharmacokinetic behaviour of the epilepsy drug valproic acid with an on-line breath sampler interfaced to a electrospray source enabling the exhaled-breath concentration of valproic acid, and its metabolites/catabolites, to be tracked continuously with the result that the elimination profile of the drug was described in a non-invasive manner. ${ }^{24}$ The sensitivity and general applicability of ESI techniques for VOC analysis continue to be developed for a variety of mass spectrometer interfaces, and estimates for limits-of-detection (LOD) continue to be reported at ever diminishing levels'; recently claimed to be in the order of $28 \mathrm{fmol} \mathrm{s}^{-1}$ for cocaine. $^{22}$ A study with 6 probe VOCs (methyl decanoate, octan-3-one, 2-ethylhexanoic acid, 1,4-diaminobutane, dimethyl methylphosphonate, and 2,3-butanediol), using a triple quadrupole mass spectrometer highlighted the importance of the operating parameters of the ionisation sources on the sensitivity of the analytical response with some indication that detection in the $\mathrm{ppq}_{v}$ to $\mathrm{ppt}_{\mathrm{v}}$ range was attainable. ${ }^{23}$ Such developments suggest that the limiting factors for trace-quantitation of VOC by ESI-MS are as likely to be associated with the challenge of generating VOC concentrations, reproducibly, at ppt(v) and lower, as with the design and optimisation of the ionisation and mass-spectrometric processes.

It is helpful to note that GC-MS analysis of human breath has revealed a chemically complicated mixture with hundreds of VOC routinely isolated over approximately fourorders of magnitude of concentration, and many of these compounds may only be detected if preconcentration approaches are adopted. ${ }^{25}$ There are a number of strategies for accomplishing preconcentration of volatiles before mass spectrometric analysis including thermal desorption (TD), ${ }^{25-27}$ solid-phase microextraction (SPME) ${ }^{28}$ and needle trap devices (NTD). ${ }^{29}$ These technologies have been used to take samples in clinical settings in conjunction with portable instrumentation. ${ }^{29}$ Thermal desorption with coldtrapping followed by recovery by ballistic heating (referred to as two-stage thermal desorption) of VOC retained within adsorbent media is used widely with GC-MS instrumentation attaining $10^{4}$ to $10^{5}$ enrichment. ${ }^{25-27}$ As well as the advantages of preconcentration, the adsorbent tubes used in TD approaches are portable, enabling large numbers of of breath samples to be taken at remote locations, and then returned to the laboratory for analysis. VOC trapped on sampling adsorbents are stable enough to be stored prior to analysis, although exactly for how long has yet to be established. Importantly, adsorbent tubes enable sterile single use and clinically safe sampling 
systems to be used to collect exhaled breath ${ }^{30}$ at the bedside, within the clinic or in the community. Previously the analysis of exhaled breath samples from human volunteer participants using a thermal desorption system hyphenated to a Waters Synapt HDMS ion-mobility mass spectrometer was described. ${ }^{31}$ With typical analysis times of 12 minutes per sample claimed, the advantages of eliminating a lengthy GC separation stage (analysis times typically up to $90 \mathrm{~min}$ ) for in-clinic applications were self-evident.

In the current work the ionisation efficiency of the low concentration VOC components recovered by thermal desorption from sampled breath was studied. Two factors were identified as important in this regard: the ubiquitous presence of siloxanes in thermal desorption profiles from breath samples ${ }^{30}$ and the mixing of the thermal desorption profile into the ESI-source. Siloxanes sequester charge and supress analyte ion formation in the ESI source, and are a significant confounding factor in the development of targeted TD-ESI-MS assays for breath analysis. An open SESI interface is at risk from disruption from background contaminants and is difficult to set up to ensure the reproducible control of all the components in the SESI-system.

Two developments were studied: a pre-separation stage with isothermal gas chromatography in the transfer line from the thermal desorption unit to isolate confounding siloxanes; and, a constrained SESI-source to ensure reproducible mixing and isolation of the SESI-region from background contamination. In-vitro studies with 5nonanone; a breath metabolite, enabled analytical figures-of-merit to be estimated before the comparative responses to 5-nonanone, benzaldehyde and 2-butanone in breath samples were evaluated. Finally, the feasibility of using TD-SESI-MS for targeted breath profiling was examined through the responses of this methodology to breath metabolites, previously indicated as potentially important ${ }^{32-34}$ candidates as markers of disease

\section{Methods and Materials}

Ethics, volunteer participants and breath samples

This research was undertaken in accordance with the ethical principles of Good Clinical Practice and the Declaration of Helsinki. The breath samples were collected using a protocol approved by the local ethics advisory committee (Ref: Loughborough University G09-P5) from volunteer participants who gave informed consent. 
For the evaluation studies $202.5 \mathrm{dm}^{3}$ exhaled distal breath samples were taken consecutively from a female participant at the Centre for Analytical Science at Loughborough University Chemistry Department. The 17 breath samples for the target compound evaluation trial were taken at the acute asthma clinic at Glenfield Hospital.

\section{Electrospray source modification}

The electrospray ionisation source of a ToFMS (Waters Synapt HDMS Waters, Manchester, UK) was modified to incorporate the outlet of a transfer line from a thermal desorption unit (Markes UNITY 1 Markes International, Rhondda Cynon Taff, UK). Reference experiments, using the configuration of the previous study were performed using the set-up shown in Figure1.31 The lockspray assembly was removed and the interlock switches were defeated to allow insertion of a $0.25 \mathrm{~mm}$ o.d. deactivated fused silica capillary from the transfer line into the source region; optimisation studies indicated that optimum sensitivity was achieved with the outlet capillary fixed at approximately $5 \mathrm{~mm}$ from the tip of the electrospray probe.

The constrained electrospray design is also shown in Figure1. In this configuration the lockspray assembly was also modified as previously described. A $25 \mathrm{~mm}$ length of stainless steel tubing ( $8 \mathrm{~mm}$ o.d., wall thickness $1.5 \mathrm{~mm}$ ) was aligned axially with the skimmer-cone inlet of the mass spectrometer and fitted so that the stainless steel tube was held in contact with the mass spectrometer sample cone to form a shroud to promote efficient mixing of the electrospray and thermally desorbed VOCs. The electrospray probe was axially aligned with the tube interface, spraying towards the mass spectrometer inlet cone, with the electrospray tip placed approximately $5 \mathrm{~mm}$ from the entrance of the tube. A $1 \mathrm{~mm}$ diameter hole in the tube interface centred approximately $1 \mathrm{~cm}$ from the electrospray inlet end allowed the capillary from the transfer line to be inserted radially to a depth of approximately $1 \mathrm{~mm}$ into the constrained ionisation source.

The transfer line from the thermal desorption unit was replaced with $1.5 \mathrm{~m}$ of $0.25 \mathrm{~mm}$ i.d. chromatography column with a $0.25 \mu \mathrm{m}$ thick $5 \%$ phenyl, $95 \%$ methyl stationary phase of (DB-5MS GC column Agilent, Milford, US). This short length of column provided the pre-separation needed to retard the desorption profiles of the larger siloxanes, thereby supressing charge sequestration, and consequently enhancing further the sensitivity of the subsequent responses. 


\section{Reagents and materials}

A 1 \% (V/v) methanoic (HPLC grade, Fisher Scientific, Loughborough, UK) solution of 5nonanone (Sigma-Aldrich, Poole, UK) was prepared for the in-vitro characterisation study. The thermal desorption sample tubes were packed with Tenax®-Carbograph1 TD (Markes International, Swansea, UK: part number - C2-AXXX-5032). Thermal desorption tubes were prepared by conditioning them at $310^{\circ} \mathrm{C}$ for $1 \mathrm{hr}$ with a $100 \mathrm{~cm}^{3} \mathrm{~min}^{-1}$ flow of helium before sealing them. Breath Samples were collected using an adaptive breath sampler 25 to collect $2.5 \mathrm{dm}^{3}$ of exhaled distal air onto the thermal desorption sample tubes. The adaptive breath sampler was described previously by Basanta et. al. ${ }^{25}$. Briefly, breaths were monitored using an integrated pressure sensor, the output of which was processed using analogue to digital conversion. The digital feed was input into a virtual instrument operating under LabviewTM that controlled two electrofluidic micro-valves. These valves ensured that a high-precision sampling pump (Escort Elf, MSA, Pittsburgh, USA), operating at a sample-flow of $1 \mathrm{dm} 3 \mathrm{~min}-1$, was maintained at a constant operating flow throughout sample acquisitions. Digital signal processing was used to continuously analyse the breathing profile and control the switching signals to ensure that the portion of the exhaled breath corresponding to the distal airways was reproducibly sampled from each breath during the sampled period.

\section{TD-ESI-MS}

Table 1 summarises the operational parameters of the instrument systems. The thermal desorption was operated without a split with a $1 \mathrm{~min}$ pre-purge at a temperature of $300^{\circ} \mathrm{C}$ for 5 min to a cold trap maintained at $-10^{\circ} \mathrm{C}$ that concentrated the thermally desorbed volatiles. Injection was by ballistic heating of the cold-trap at $50{ }^{\circ} \mathrm{C} \mathrm{s}^{-1}$ to 300 ${ }^{\circ} \mathrm{C}$; maintained for $3 \mathrm{~min}$. The transfer line was maintained at $120^{\circ} \mathrm{C}$, and the desorption flow through the cold-trap and transfer-line was $5 \mathrm{~cm}^{3} \mathrm{~min}^{-1}$ of purified helium.

The mass spectrometer (Synapt HDMS Waters, Manchester, UK) was operated with the ion mobility function disabled. All experiments were performed in the positive ion mode over a mass range of $\mathrm{m} / \mathrm{z} 50$ to $\mathrm{m} / \mathrm{z} 500$. 


\section{Results and Discussion}

The effect of a constrained source.

The constrained source (Figure1) was implemented to contain and enhance the mixing of the electrospray and the thermal desorption plumes. The utility of doing this was characterised with a series of experiments involving the recovery of ca. $1 \mu \mathrm{g}$ of 5 nonanone from a conditioned thermal desorption tube (such a loading is equivalent to taking a $2.5 \mathrm{dm}^{3}$ breath sample with a distal concentration of $70 \mathrm{ppb}_{\mathrm{v}}$ ). The 5-nonanone was spiked on to the adsorption tube with a $0.1 \mu$ injection of $1 \%(v / v) 5$-nonanone in methanol. The insert in Figure 2 compares the responses observed with the constrained SESI source against an open SESI configuration. The maximum intensity response increased from 1.7 kcount to 6.1 kcount (an increase of 3.7 times) and the integrated desorption profile increased from 23 kcount.s to 106 kcount.s (a 4.6-fold enhancement).

The proof-of-concept study for the constrained source compared the 5-nonanone $\mathrm{m} / \mathrm{z}$ $143\left(\mathrm{MH}^{+}\right)$calibration and limits-of-detection for the constrained SESIsource with preseparation, against the open SESI source without pre-separation. The calibration was determined from the linear regression of $\mathrm{m} / \mathrm{z} 143\left(\mathrm{MH}^{+}\right)$integrated ion intensity against analyte masses $\left(m_{i}\right)$ over the range $100 \mathrm{pg}$ to $200 \mathrm{ng}$ loaded on tube; equivalent to a 2.5 $\mathrm{dm}^{3}$ breath sample of exhaled concentrations from ca.7 ppt to $13 \mathrm{ppb}_{\mathrm{v}}$. Equation 1 is the calibration obtained for the open SESI source configuration, and Equation 2 is the comparison for the constrained source with pre-separation; note that the values in the square bracket denote the $95 \%$ confidence limits of the parameters.

$$
\begin{gathered}
I(\text { count })=138[ \pm 207](\text { count })+15[ \pm 4.1]\left(\text { count ng }^{-1}\right) \times m_{i}(\mathrm{ng}) ; R^{2}=0.98 \\
I(\text { count })=-64[ \pm 223](\text { count })+55[ \pm 5.33]\left(\text { count ng }^{-1}\right) \times m_{i}(\mathrm{ng}) ; R^{2}=0.995
\end{gathered}
$$

The limit of detection 35 for 5-nonanone for the open source was estimated to be not more than $56 \mathrm{ng}$ on tube mass, equivalent to a breath concentration of ca. $4 \mathrm{ppb}_{\mathrm{v}}$ in a $2.5 \mathrm{dm}^{3}$ breath sample, and $14.3 \mathrm{ng}$ on tube mass (967 ppt $\mathrm{p}_{\mathrm{v}}$ ) for the constrained SESI source with pre-separation. The difference from 0 of the negative intercept for the closed source was not statistically significant, however it may be indicative of non-linear responses at low concentrations, and this is consistent with the reduction in the ionneutral collision frequency with a falling concentration combined with the constant residence time (ventilation rate) of the neutrals in the constrained ionisation volume. In 
this instance the constrained SESI source with separation was 3.7 times more sensitive with a limit of detection about a quarter as low again as that of the open SESI source without pre-separation. However such an evaluation does not address the confounding presence of siloxanes in breath samples with their high water loadings, to evaluate this phenomenon 20 breath samples taken sequentially from a volunteer participant were analysed.

Pre-separation to inhibit siloxane charge sequestration.

Siloxanes have been found to be ubiquitous contaminants in clinical breath samples. 30 and their high proton affinity results in charge sequestration and suppression of analyte ion formation. ${ }^{31}$ Indeed the cyclosiloxanes decamethylcyclopentasiloxane $(\mathrm{m} / \mathrm{z} 371.0817$ $\left.[\mathrm{M}+\mathrm{H}]^{+}\right)$and dodecamethylcyclohexasiloxane $\left(\mathrm{m} / \mathrm{z}\right.$ 445.1040 $\left.[\mathrm{M}+\mathrm{H}]^{+}\right)$dominated ESI mass spectra acquired from breath samples reported previously. ${ }^{31}$ (They are also a significant feature in GC-MS chromatograms obtained from electron impact ionization, where they have been used as secondary retention indexing standards. ${ }^{30}$ ) Substituting the deactivated capillary transfer line with $1.5 \mathrm{~m}$ of a $0.25 \mu \mathrm{m}$ film thickness DB-5MS (5 $\%$ phenyl, $95 \%$ methyl) GC-column reduced the siloxane-based interference of the signal. With the transfer line temperature maintained at $120^{\circ} \mathrm{C}$, it was possible to retard elution of decamethylcyclopentasiloxane and dodecamethylcyclohexasiloxane through the transfer line relative to other breath metabolites and hence enhance the ion production of the more volatile breath metabolites. Figure 2 compares mass selected thermal desorption profiles of breath samples obtained from open and constrained sources with and without a chromatographically active transfer line. With an open source and no preseparation in the transfer line an intense peak for the total ion signal (42 kcounts), with some minor features on its tail, was observed. Introducing a constrained SESI source with pre-separation in the transfer line resulted in several poorly resolved bands and an increase in the analysis time of approximately $3 \mathrm{~min}$. Predictably, with the recovered VOC separated into distinct bands the maximum intensity of the total ion desorption signal (3.3 kcounts) reduced significantly. Nevertheless the signal-to-noise relationships for individual ions increased markedly, with a corresponding reduction in the siloxane interference intensities. For an open ESI with no pre-separation the maximum intensity of the dodecamethylcyclohexasiloxane $(\mathrm{m} / \mathrm{z}$ 445.2) was ca. 18 kcounts, in contrast with a constrained source with pre-separation this reduced to 422 counts. Similarly the maximum desorption profile intensity for decamethylcyclopentasiloxane $(m / z \quad 371.2)$ 
reduced from 6 kcounts (open SESI no pre-separation) to 337 counts (constrained SESI with pre-separation). The reduction in the signals for the siloxane interferences was accompanied by increases in the signals for endogenous VOC. The example in Figure 2 shows that the 5 -nonanone $(m / z$ 143.15) desorption profile obtained with the open SESI without pre-separation from breath samples was at best a discernable perturbation in the base-line, contrasted to a signal of 25 counts from the constrained source with preseparation for a similar sample. Acetone $(m / z 59.05)$ showed a similar enhancement with 69 counts increasing to 415 counts and cyclohexenone and/or dimethylfuran $(\mathrm{m} / \mathrm{z}$ 97.07) increased from 27 counts to 86 counts. These observations support the proposition that the presence of high-proton affinity siloxanes result in charge sequestration and signal suppression for many exhaled breath metabolites.

Comparison of the mass spectra obtained from the two systems, Figure 3, supports the claim that constrained SESI enhances ionisation and pre-separation suppresses charge sequestration by siloxanes. The mass spectra for the open SESI source with no preseparation show the significant presence of siloxanes see $m / z 355, m / z 371, m / z 429$ and 445. In contrast these features are strongly supressed with a constrained SESI source with pre-separation. The features at $\mathrm{m} / \mathrm{z} 355$ and 371 are reduced by $98 \%$, 2719 counts to 63 counts and 3413 counts to 74 counts respectively, while the interferent at $\mathrm{m} / \mathrm{z} 429$ was reduced from 529 counts to 34 counts, a 95.6\% reduction, and finally at $m / z 445$ the signal was reduced from 1360 to 108 a $92.8 \%$ reduction. The effect in the chemical information content, (or chemical space) within the desorption profile may be discerned from the cumulative distribution function (CDF), also shown in Figure 3. The mass spectra in Figure 3 were filtered to remove noise, so signals below the limit-of-detection were removed and a histogram of peak intensity vs. $m / z$ was produced.The resultant CDF for the open SESI-source showed distinct step-like features associated with the siloxane species, and in contrast to the constrained SESI with preseparation, there are $m / z$ ranges where the contribution to the CDF was supressed. In fact from about $\mathrm{m} / \mathrm{z} 200$ upwards the contribution was almost exclusively due to siloxanes species accounting for ca. 1/3 of the total response. The constrained source generated responses across the whole mass range and the increase in analytical space that resulted may be discerned. Further study of the intensity of the ions observed from the two sources reveals that the constrained SESI source with pre-separation resulted in significant increases in the number of ions observed at low concentrations, see Figure 4. In Figure 4 the mass spectra in Figure 3 were characterised in terms of the total number 
of analytical features (peaks) observed over a given normalised intensity range. For $97.5 \%$ of the normalised intensity range from 0.025 upwards to 1 the distributions of the number of mass spectral peaks observed was essentially the same for both approaches. However what was clear was that the greatest number of analytical features were to be observed at the lowest intensities. Significantly more analytical features were observed using the constrained SESI source with pre-separation. The open SESI without preseparation yielded 260 mass spectral peaks, of which 179, (69\%) were present at a normalised intensity of equal to or less than 0.025 . In contrast the constrained source yielded 541 peaks, with 448 (83\%) present at intensities equal to or less than a normalised intensity of 0.025 .

\section{Reproducibility}

Reproducibility measurements were based on 20 replicate breath samples collected sequentially from a healthy volunteer. 10 samples selected randomly were analysed by each of the techniques and the reproducibility compared. Figure 5 compares responses obtained for 5-nonanone, $(\mathrm{m} / \mathrm{z}$ 143.03), benzaldehyde $(\mathrm{m} / \mathrm{z} 107.12)$ and 2-butanone $(m / z$ 73.08), and in this figure the selected-ion thermal desorption profiles are normalised to the group average enabling the differences in signal to noise and $95 \%$ confidence limits to be evaluated. The enhancement in sensitivity and reproducibility obtained from the constrained source with pre-separation is evident. The relative standard deviations (RSD) obtained for 5-nonanone, benzaldehyde and 2-butanone from the constrained source with pre-separation were $28 \%, 16 \%$ and $14 \%$ respectively, and the corresponding values for the open source were that 5-nonanone was not detected, with RSDs of 39\% for benzaldehyde and $31 \%$ for 2-butanone.

Clinical pilot

The final test undertaken was a pilot study to assess the feasibility of adopting this approach for targeted breath profiling. 17 exhaled breath samples were obtained from volunteers attending or working at a severe asthma clinic (Glenfield Hospital, Leicester, UK). 10 samples were analysed by TD-SESI-ToFMS with a constrained source with preseparation and 7 were analysed with TD-SESI-ToFMS with an open source and no preseparation. $11 \mathrm{VOC}$, reported previously in breath profiling studies of respiratory disease ${ }^{36-39}$, were selected as target compounds and the thermal desorption profiles were screened in the positive mode for protonated product ions. 
Table 2 is a summary of the endogenous breath VOC identification from the two sets of data. It was not always possible to obtain accurate mass measurements ( $<15 \mathrm{ppm})$ in the absence of a low $\mathrm{m} / \mathrm{z}$ lock mass ion. The constrained source with pre-separation enabled 1-hydroxyacetone, 3-buten-2-one, and acetone to be observed in all samples. 2,3-Butanedione, butanone, pentanal, benzaldehyde, isoprene, and trimethylamine were observed in 9 of the samples with dimethylsulfide present $80 \%$ of the time in 8 samples and finally dimethylamine was observed in 3 samples, 30\%. In contrast acetone and dimethylamine were observed in all the samples analysed with the open source with no pre-separation. 1-Hydroxyacetone, butanone and isoprene were observed in 6 of the 7 samples (86\%). Benzaldehyde (71\%), dimethylsulfide (71\%), 3-buten-2-one (57\%), trimethylamine (57\%), 2,3-butandione (43\%) and pentanal (43\%) all appeared to be observed less frequently compared to the constrained source with pre-separation.

In those instances when it was possible to assign ions on the basis of accurate mass (to within $15 \mathrm{ppm}$ of the monoisotopic mass), the thermal desorption profile was integrated and the mean responses in Table 2 indicate that the constrained source with preseparation resulted in a higher proportion of accurate masses and more sensitive responses.

\section{Conclusions}

Constraining the dispersal of the analyte plume and promoting mixing into the electrospray ion jet combined with a pre-separation stage results in larger numbers of VOC being observed in exhaled breath. Further the responses appear to be more sensitive with a higher reproducibility.

The significant increase in the numbers of VOC observed with the constrained SESI with pre-separation emphasises and that changes in sampling and/or ionisation and/or detection are likely to exert profound effects on the quantity of available metabolic information recovered during a study. Just how many VOC are present in a typical breath sample and at what concentrations has yet to be determined. When ambient ionisation approaches are adopted the potential of charge transfer processes within the ionisation source to bias responses to compounds with higher proton affinity needs to be addressed; the increase in proportion of dimethylamine observations from the open source for example, Table 2. The central importance for systemic control and self-critical approaches to quality assurance in metabolomic-based breath profiling studies are highlighted here. Certainly, reporting on the numbers and distribution of analytical 
features associated with breath profiling methods would assist in data evaluation, and consolidation of research findings. This is particularly important with ambient ionisation techniques where selective ionisation and ion suppression effects can have a dramatic effect on the mass spectral response.

This study indicates that faster targeted analysis of trace VOC in breath is possible by TDSESI-MS, with the use of a constrained electrospray plume in conjunction with preseparation to isolate the breath volatiles from siloxane contaminants. This technique appears to have potential as a rapid screening method for biomarkers in breath in the $\operatorname{ppt}(\mathrm{v})$ to $\mathrm{ppb}(\mathrm{v})$ range with analysis times of ca. $12 \mathrm{~min}$, and although this study addresses only a small number of analytes from a limited number of participants, this does demonstrate proof-of-priciple andmerits further development. The next challenge is to develop the ionisation systems for VOC that do not form ions in a SESI source, so that these species can be included in this approach.

\section{Acknowledgments}

The authors gratefully acknowledge the Daphne Jackson Trust and the sponsorship of EPSRC and Loughborough University for the support to Dr. Modupe Jimoh. Dr. Cristina Guallar Hoyes was supported jointly by funding from Astra Zeneca and Loughbrough University. The in-clinic studies were supported by the National Institute for Health Research (NIHR) Leicester Respiratory Biomedical Research Unit. The views expressed are those of the author(s) and not necessarily those of the NHS, the NIHR or the Department of Health

\section{References}

$1 \quad$ Yamashita, M; Fenn, J.B. J. Phys. Chem. 1984, 117, 4451

2 Whitehouse, C.M; Levin, F; Meng, C.K; Fenn, J.B. Proceedings of the 34th ASMS Conference on Mass Spectrometry and Allied Topics, Denver, 1986, pp507

3 Chen, Y.H; Hill, H.H; Wittmer, D.P, J. Microcolumn Separations, 1994, 6, 1994

4 Wu, C; Siems, W.F; Hill, H.H, Anal. Chem., 2000, 72, 396

5 Martinez-Lozano, P; Fernández de la Mora, J; Anal. Chem, 2008, 80, 8210 
6 Martinez-Lozano, P; Zingaro, L; Finiguerra, A; Cristoni, S, J. Breath Res, 2011, 5, DOI: 10.1088/1752-7155/5/1/016002

7 Chen, H; Venter, A; Cooks, R.G, Chem. Commun., 2006, 2042

8 Devenport, N.A.; Sealey, L.C.; Alruways, F.H.; Weston, D.J.; Reynolds, J.C.; Creaser, C.S., Anal. Chem. 2013, 85, 6224.

9 Smith, R.W.; Reynolds J.C.; Lee, S-L.; Creaser, C.S., Anal Methods, 2013, 5, 3799.

10 Lee, C-Y; Shiea, J, Anal. Chem, 1998, 70, 2757-2761.

11 Brenner, N, Haapala. M, Vuorensola. K, Kostiainen. R, Anal. Chem, 2008, 80, 8334-8339.

12 Miekisch. W, Schubert. J.K, Trends in. Analytical. Chemistry., 2006. 25,: 665-673.

13 Amann. A, Spanel. P,. Smith. D,, Breath Analysis: The Approach Towards Clinical Applications, Mini Rev. Med. Chem, 2007,. 7, (2007)115.

14 Dummer J, Storer M, Swanney M, McEwan. M, Scott-Thomas. A, Bhandari. S, Chambers. S, Dweik. R, Epton. M, Trends in Analytical Chemistry, 2011, 30 (7), 960-967

15 Amann. A, Smith. D,, Volatile Biomarkers, 1st Edition, Non-Invasive Diagnosis in Physiology and Medicine, Elsevier 2013, ISBN: 9780444626134

16 Das. M.K, Bishwal. S,C, Das. A, Dabral. D, Varshney. A, Badireddy. V.K, Nanda. R, Anal. Chem, 2014, 86, 1229-1237

17 Trefz. P, Schmidt. M, Oertel. P, Obermeir. J, Brock. B, Kamysek. S, Dunkl. J, Zimmermann. R, Schubert. J.K, Miekisch. W, Anal. Chem, 2013, 85(21), 1032110329

18 Storer. M, Dummer. J, Sturney. S, Epton. M, Current Analytical Chemistry, 2013, 9(4), 576-583 


\title{
ANALYSIS OF HUMAN BREATH SAMPLES USING A MODIFIED THERMAL DESORPTION - GAS CHROMATOGRAPHY ELECTROSPRAY IONIZATION INTERFACE.
}

\author{
${ }^{1}$ James C. Reynolds, ${ }^{1}$ Modupe A. Jimoh, ${ }^{1}$ Cristina Guallar-Hoyas, ${ }^{1}$ Colin S. Creaser, \\ 2Salman Siddiqui, ${ }^{1}$ C.L. Paul Thomas \\ ${ }^{1}$ Centre for Analytical Science, Department of Chemistry, \\ Loughborough University, Ashby Road, Loughborough, LE11 3TU UK \\ 2NIHR Leicester Respiratory Biomedical Research Unit \\ Glenfield Hospital, Groby Road, Leicester, LE3 9QP
}

Tables

Table 1. Summary of the mass spectrometer operating parameters

\begin{tabular}{|c|c|c|c|c|}
\hline Parameter & \multicolumn{2}{|c|}{ Open Source } & \multicolumn{2}{|c|}{ Constrained Source } \\
\hline \multicolumn{5}{|l|}{ Thermal desorption } \\
\hline Desorption flow & 5 & $\mathrm{~cm}^{3} \mathrm{~min}^{-1}$ & 5 & $\mathrm{~cm}^{3} \min ^{-1}$ \\
\hline Sample-trap leak-test & 0 to -1 & $\min$ & 0 to -1 & $\min$ \\
\hline Sample trap pre-purge & 1 to 2 & $\min$ & 1 to 2 & $\min$ \\
\hline Sample trap desorption & 2 to 6 & $\min$ & 2 to 6 & $\min$ \\
\hline Desorption temperature & 300 & ${ }^{\circ} \mathrm{C}$ & 300 & ${ }^{\circ} \mathrm{C}$ \\
\hline Desorption split & Splitless & & Splitless & \\
\hline Cold trap temperature & -10 & ${ }^{\circ} \mathrm{C}$ & -10 & ${ }^{\circ} \mathrm{C}$ \\
\hline Cold-trap pre-purge & 6 to 7 & $\min$ & 6 to 7 & $\min$ \\
\hline Cold-trap secondary desorption & 7 to 10 & $\min$ & 7 to 10 & $\min$ \\
\hline Cold-trap heating rate & 50 & ${ }^{\circ} \mathrm{C} \mathrm{s}^{-1}$ & 50 & ${ }^{\circ} \mathrm{C} \mathrm{s}^{-1}$ \\
\hline Cold-trap desorption temperature & 300 & ${ }^{\circ} \mathrm{C}$ & 300 & ${ }^{\circ} \mathrm{C}$ \\
\hline Cold-trap cooling & 10 to 11 & $\min$ & 10 to 11 & $\min$ \\
\hline Sample-trap cooling & 6 to 10 & $\min$ & 6 to 10 & $\min$ \\
\hline \multicolumn{5}{|l|}{ Mass spectrometer } \\
\hline Capillary voltage & 3 & $\mathrm{kV}$ & 3 & $\mathrm{kV}$ \\
\hline Sample cone voltage & 20 & V & 20 & V \\
\hline Extraction cone voltage & 4 & V & 5 & V \\
\hline ESI solvent flow rate & 5 & $\mu \mathrm{l} \min ^{-1}$ & 5 & $\mu \mathrm{l} \min ^{-1}$ \\
\hline Desolvation temperature & 200 & ${ }^{\circ} \mathrm{C}$ & 120 & ${ }^{\circ} \mathrm{C}$ \\
\hline Source Temperature & 120 & ${ }^{\circ} \mathrm{C}$ & 120 & ${ }^{\circ} \mathrm{C}$ \\
\hline Desolvation gas flow & 500 & $\mathrm{dm}^{3} \mathrm{hr}^{-1}$ & 100 & $\mathrm{dm}^{3} \mathrm{hr}^{-1}$ \\
\hline Cone gas flow & 0 & $\mathrm{dm}^{3} \mathrm{hr}^{-1}$ & 20 & $\mathrm{dm}^{3} \mathrm{hr}^{-1}$ \\
\hline
\end{tabular}




\begin{tabular}{|c|c|c|c|c|c|c|c|}
\hline \multirow{2}{*}{ Metabolite } & \multirow[b]{2}{*}{$M$} & \multicolumn{3}{|c|}{$\begin{array}{l}\text { Closed source with pre- } \\
\text { separation }\end{array}$} & \multicolumn{3}{|c|}{$\begin{array}{l}\text { Open source no pre- } \\
\text { separation }\end{array}$} \\
\hline & & $\bar{M} ; 95 \%$ & a\% & $\begin{array}{c}\bar{R} \\
(\mathrm{AU})\end{array}$ & $\bar{M} ; 95 \%$ & b\% & $\begin{array}{c}\overline{\boldsymbol{R}} \\
(\mathrm{AU})\end{array}$ \\
\hline 1-Hydroxyacetone & 75.045 & $74.9 ; 0.2$ & 100 & 2.89 & $75.042 ; 0.001$ & 86 & 1.6 \\
\hline 3-Buten-2-one & 71.05 & $71.05 ; 0.01$ & 100 & 4.92 & $71.047 ; 0.001$ & 57 & 3.8 \\
\hline 2,3-Butanedione & 87.045 & $87.044 ; 0.001$ & 90 & 7.58 & $87.042 ; 0.001$ & 43 & 3.0 \\
\hline Butanone & 73.065 & 73.06; 0.01 & 90 & 7.28 & 73.06; 0.01 & 86 & 3.2 \\
\hline Pentanal & 87.081 & $87.06 ; 0.01$ & 90 & & 87.08; 0.01 & 43 & \\
\hline Benzaldehyde & 107.05 & $107.06 ; 0.01$ & 90 & 6.12 & 107.048; 0.001 & 71 & 4.9 \\
\hline Acetone & 59.05 & 59.049; 0.001 & 100 & 9.79 & $59.047 ; 0.001$ & 100 & 2.4 \\
\hline Isoprene & 69.070 & $69.070 ; 0.001$ & 90 & 10.37 & 69.068; 0.001 & 86 & 14 \\
\hline Trimethylamine & 60.081 & $60.06 ; 0.03$ & 90 & 0.39 & 60.073; 0.01 & 57 & \\
\hline Dimethylsulfide & 63.027 & $63.01 ; 0.03$ & 80 & & 62.999; 0.002 & 71 & \\
\hline Dimethylamine & 46.066 & $46.065 ; 0.001$ & 30 & 1.26 & $46.063 ; 0.001$ & 100 & \\
\hline
\end{tabular}

Note:

$M$ : nominal mass, $\overline{\boldsymbol{M}}$; 95\%: mean observed mass; $95 \%$ confidence limit, \% percentage of samples found with target compound in, a 10 samples with the constrained source, and b 7 samples with the open source, $\bar{R}$ (AU) mean response (au: arbitrary units) from those analyses where the observed mass was within $50 \mathrm{ppm}$ of the nominal mass.. 

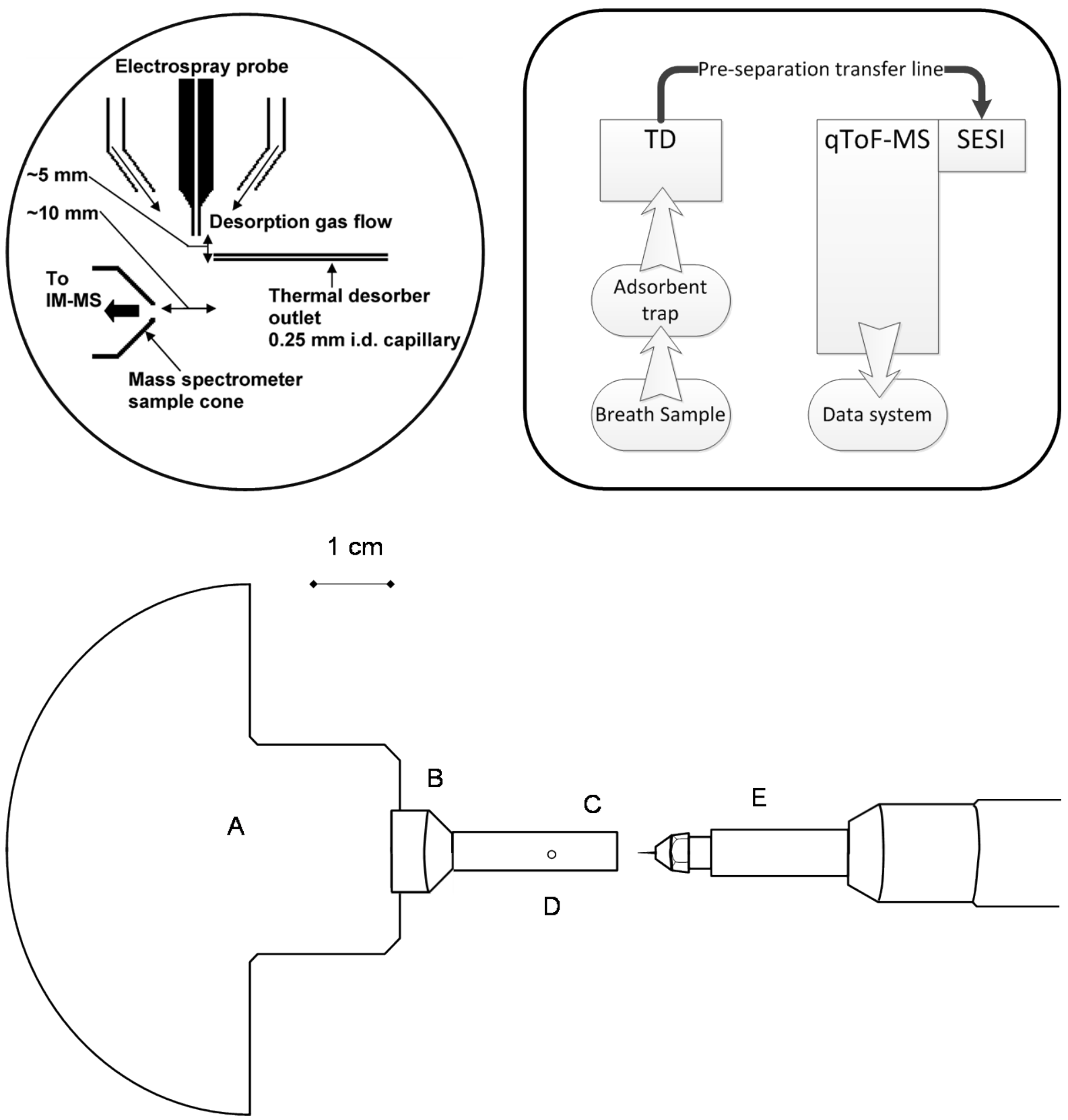

Figure 1. Top left insert an unconstrained thermal desorption SESI interface. (Reprinted with permission from Reynolds, J.C; Blackburn, G.J; Guallar-Hoyas, C; Moll, V.H; Bocos-Bintintan, V; Kaur-Atwal, G; Howdle, M.D; Harry, E.L; Brown, L.J; Creaser, C.S; Thomas, C.L.P, Anal. Chem., 2010, 82, 2139. Copyright (2010) American Chemical Society)

Top right a functional schematic of the experimental set up

Bottom. Constrained source showing the mass spectrometer inlet manifold $(A)$ and mass spectrometer inlet cone (B)modified by attaching a $2 \mathrm{~cm}$ length of $8 \mathrm{~mm}$ diameter stainless steel tubing (C), wall thickness $1.5 \mathrm{~mm}$, with a $1 \mathrm{~mm}$ hole to enable the transfer line from the thermal desorption unit to be inserted ca. $1 \mathrm{~cm}$ from the entrance to the inlet tube (D). The electrospray probe was offset by ca. $5 \mathrm{~mm}$ and axially aligned with the stainless steel tube $(\mathrm{E})$. 


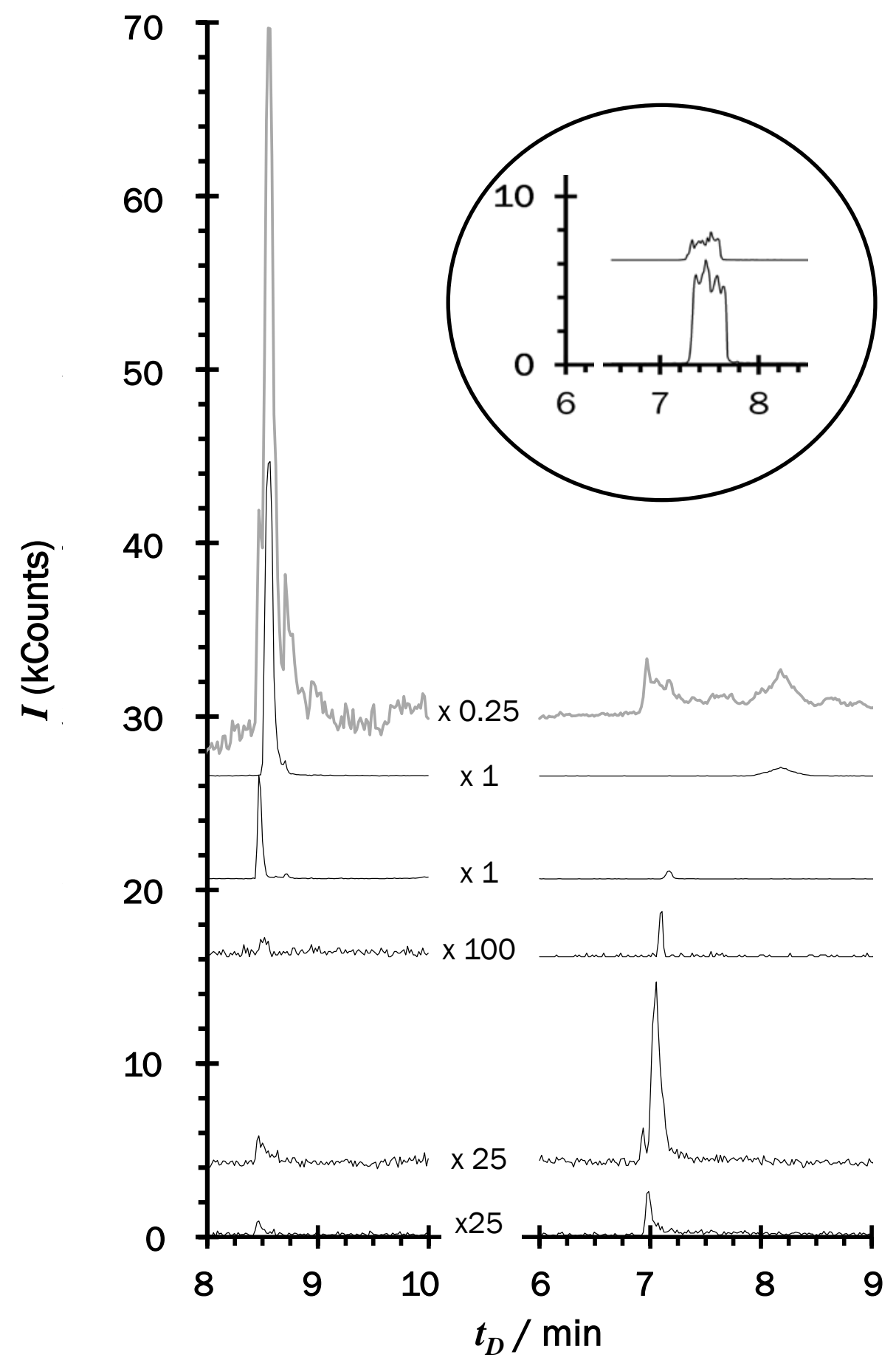

Figure 2 Mass selected thermal desorption profiles showing the effect on sensitivity of introducing a constrained source with pre-separation. Inset: selected ion desorption profile $[\mathrm{M}+\mathrm{H}]^{+}$of 5-nonanone standard, indicating the enhancement of the ion signal from constraining the source without pre-separation. LHS traces compare open source responses without pre-separation to RHS traces showing constrained sources with a pre-separation step. Figures indicate scaling of signal. From top-to-bottom: total ion trace (x 0.25); dodecamethylcyclohexasiloxane $(\mathrm{m} / \mathrm{z}$ 445.2, x 1) decamethylcyclopentasiloxane (m/z 371.2, x1); nonan-5-one (m/z $143.15, x 100)$ acetone $(\mathrm{m} / \mathrm{z} 59.05, \mathrm{x} 25)$ and cyclohexenone $(\mathrm{m} / \mathrm{z} 97.07, \mathrm{x} 25)$. 

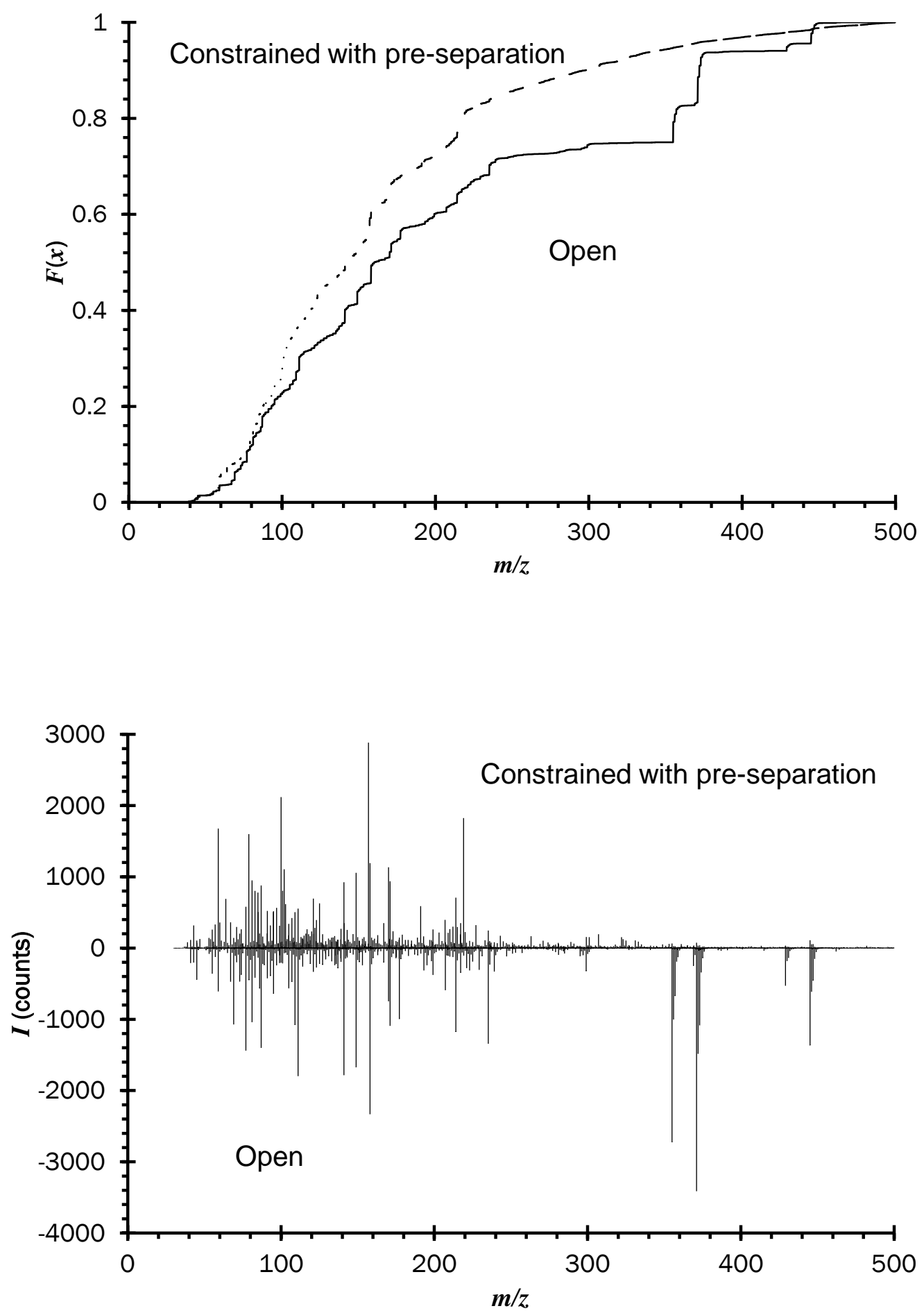

Figure 3. Mass spectra (bottom), and their cumulative distribution functions, $(F(x) v s . m / z$ from breath samples taken from the same participant, at approximately the same time illustrating the effect of constraining the SESI source with pre-separation on the resultant data. The large siloxane responses were effectively supressed, and their distortion of the cumulative distribution function eliminated. Note the increased chemical space associated with the constrained SESI source with preseparation; arising from a significant increase in number of lower intensity signals observed, see Figure 4. 


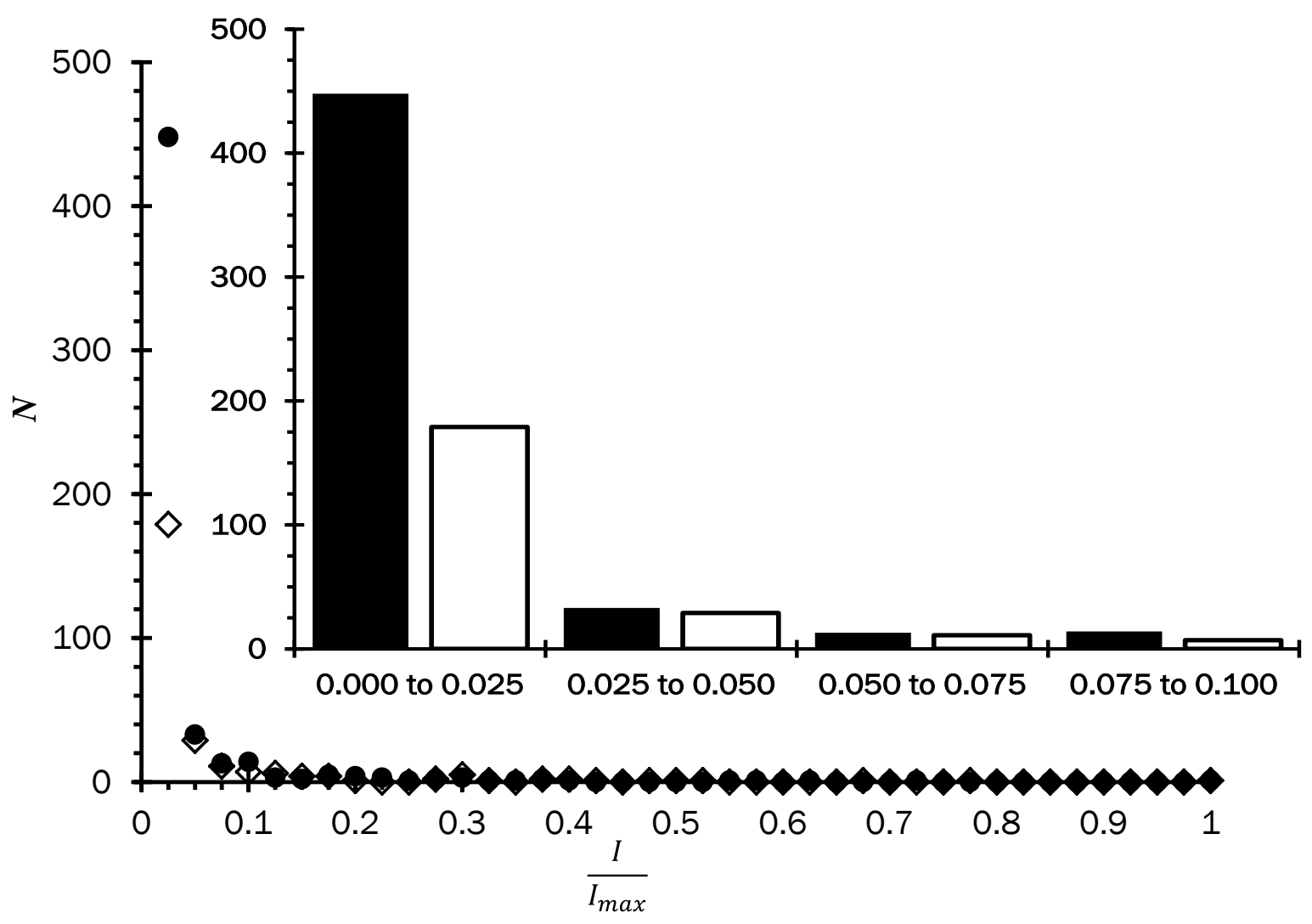

Figure 4. Distribution of responses against normalised intensity . Circles show the number of analytical features over normalised interval for the constrained SESI source with pre-separation, and open diamonds show the comparison data from the open SESI source without pre-separation. Insert, a histogram showing a close up of the first $10 \%$ of the normalised response range. Black constrained SESI with pre-separation and white open SESI without pre-separation. Using the constrained SESI source with pre-separation resulted in significantly increased numbers of low concentration VOC in exhaled breath being observed, without affecting the responses to the higher level components. 


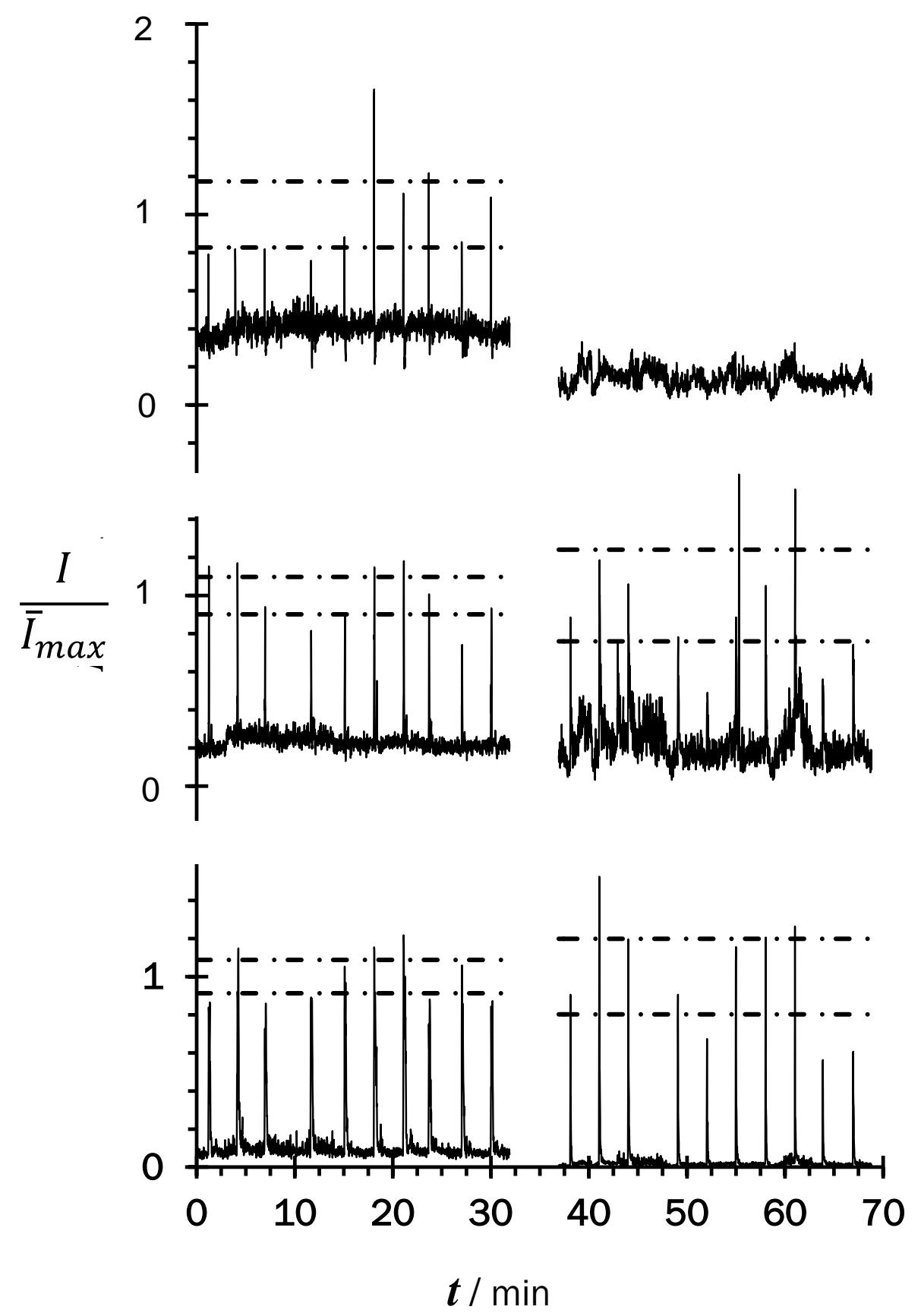

Figure 5 Composite offset selected ion thermal desorption profiles of replicate breath samples showing ion selective signal intensity $(I)$ normalised to the mean maximum intensity of the ion $\left(\bar{I}_{\max }\right)$. The $95 \%$ confidence limits are indicated with dashed lines. The left hand traces were obtained from the constrained source with pre-separation and the right from the open source. Top: 5-nonanone (143.03), middle: benzaldehyde ( $\mathrm{m} / \mathrm{z}$ 107.12), and bottom butanone $(\mathrm{m} / \mathrm{z}$ 73.08). The variations in sensitivity, and reproducibility of the responses to these three breath volatiles are evident. 